\title{
Pensar la complejidad, pensar como síntesis
}

THINKING COMPLEXITY, THINKING AS SYNTHESIS

Dr. Carlos Eduardo Maldonado (carlos.maldonado@urosario.edu.co) Facultad de Ciencia Política y Gobierno, Universidad del Rosario (Colombia)

\begin{abstract}
After a number of general considerations, without meaning in any sense a reductionist approach, this paper argues in favour of the mathematics of discrete systems and of the non-classical logics, and claims that a complex thinking both entails and crosses through those domains. Such a proposal, it is argued, has not been a general concern until to-date among the communities of complexologists. At the end, several consequences are withdrawn at understanding what truly thinking about complexity is all about.
\end{abstract}

Key words: sciences of complexity, mathematics of discrete systems, non-classical logics, synthesis, epistemology.

\section{Resumen}

Luego de varias consideraciones de orden general, y sin que se asuma en absoluto una postura reduccionista, este texto argumenta a favor de la tesis según la cual pensar la complejidad con la ciencia de punta implica considerar los modos y grados como las matemáticas de sistemas discretos y las lógicas no-clásicas contribuyen a la dilucidación de lo que es un pensar la complejidad. Estas consideraciones no han sido incorporadas en general por parte de la comunidad de complejólogos. Al final se extraen algunas de las consecuencias de lo que, consiguientemente, sea pensar la complejidad.

Palabras clave: ciencias de la complejidad, matemáticas de sistemas discretos, lógicas no-clásicas, síntesis, epistemología.

\section{Introducción}

Ante el gran público, e incluso ante complejólogos aficionados, la asociación entre pensar y pensamiento y complejidad remite casi inmediatamente a varios textos de Edgar Morin. Y por derivación a la identificación entre "el método" y el pensamiento complejo. Una asociación semejante no es necesaria e incluso resulta equívoca. El pensar no es exclusivo de ninguna escuela en ningún ámbito del conocimiento. Por tanto, con este texto me propongo elucidar una perspectiva adicional, a saber: cómo es pensar la complejidad en la perspectiva de las ciencias de la complejidad, un tema que no ha sido plenamente abordado ni elucidado tampoco del lado de los partidarios de las ciencias de la complejidad.

Ahora bien, el trabajo con sistemas, fenómenos o comportamientos de complejidad creciente no es ni evidente ni tampoco necesario. La prueba es que a pesar de la creciente masa crítica alrededor del mundo -congresos, revistas especializadas, redes de colaboración, colecciones en editoriales, por ejemplo-, los 
complejólogos aún nos encontramos lejos de ser el paradigma dominante en la ciencia. La complejidad en general siendo una postura alternativa o marginal. Pues bien, más difícil aún es la elucidación de lo que sea pensar la complejidad en general. La inmensa mayoría de los trabajos que se publican en complejidad tienen un impacto importante en numerosos dominios, y ciertamente hacen contribuciones puntuales en diversas esferas del conocimiento y el mundo, pero son generalmente minimalistas, acaso por técnicos.

Este texto argumenta en el sentido de que es indispensable un trabajo de gran envergadura, de alcances al mismo tiempo científico, social y cultural en complejidad, en fin, de alcance sintético, junto a los trabajos ampliamente dominantes de carácter técnico que se publican en complejidad. En este texto me propongo justificar esta idea y señalar las dimensiones y modos, así como los alcances y las consecuencias de la misma. La tesis que defiende este texto es que el pensar de la complejidad es esencialmente sintético. Para ello, se presentan tres argumentos. En primer lugar, se señalan históricamente los grandes momentos en los que un pensar sintético ha emergido y sus consecuencias en la historia de la humanidad. Este argumento tiene una finalidad histórica. Seguidamente, se caracteriza el modo de pensar propio de la complejidad, que es la síntesis (este es el núcleo de este artículo) y, finalmente, el tercer argumento se ocupa con las consecuencias e implicaciones teóricas y prácticas de un pensar complejo. En la conclusión sostenemos que pensar sintéticamente entraña y ha implicado un gran impulso civilizatorio en la historia de la humanidad.

\section{Modos y momentos de síntesis en la historia de la humanidad}

Contra todas las apariencias, el avance en el pensar no tiene lugar por vía acumulativa. Por el contrario, la evolución en el pensar sucede por vía de dos modos: rupturas y quiebres, y síntesis. El primero de los modos ha ocupado la atención de investigadores y teóricos, por ejemplo de la historia y la filosofía de la ciencia, y por el contrario, los avances por vía de síntesis no han ocupado tan destacado lugar en los estudios y reflexiones. Este texto se concentra, por consiguiente, en este segundo modo.

Pensar en síntesis ha constituido, sin la menor duda, una de las formas más importantes de jalonamiento en la historia del conocimiento, y siempre ha coincidido con inflexiones fundamentales en la historia del espíritu humano. Esta primera sección tiene como doble finalidad mostrar que, contra todas las apariencias, ha habido ya momentos fundamentales en los que la sociedad humana ha pensado y vivido en términos de síntesis, y con ello, al mismo tiempo, dejar en claro que es posible y cómo, por lo menos por analogía, pensar en términos de síntesis.

Sobre la base trabajos por parte de historiadores, filósofos y expertos en temas culturales (Lévêque 2012, Roberts 2010), es posible identificar cuatro momentos de un pensamiento sintético en la historia del espíritu humano. Lo fundamental, con todo, consiste en establecer que cada uno de estos cuatro momentos ha sido un impulso civilizatorio de la humanidad. Esto es, se trata de cuatro pasos o momentos en los que lo que sea la especie humana ha encontrado inflexiones singulares que han marcado, de lejos y durante mucho tiempo, a la historia restante de la humanidad. O bien, para decirlo inversamente, se trata de cuatro momentos a los que retrospectivamente, las culturas y sociedades posteriores han retornado en algún momento como alimento, sentido y plataforma para hacer posibles pasos subsiguientes. Estos cuatro momentos son: 1) alrededor del año 3000 a.e.v. (antes de la era vulgar), 2) en el siglo $\mathrm{VI}$ a.e.v. cuando se catapulta la actual civilización que se llama a sí misma Occidente, 3) hacia el año 1100 cuando tiene lugar el primer Renacimiento y que desemboca en el Quattrocento, y finalmente, 4) en nuestros días y, quiero decirlo, muy exactamente en el contexto y medio de las ciencias de la complejidad. 
Poco estudiado, el primer momento comprende la cultura Liangzhu en el delta del río Amarillo en China, el primer período Harappan en el valle de la India, que es un momento importante de pensamiento y actividad integrativa; en otra latitud, Stonehenge comienza a ser construido, comienza la civilización minoica, y paralelamente comienza la civilización cicládica; en las Américas tienen sus orígenes la civilización Norte Chico en Perú, y en términos muy genéricos, es, en general, el inicio de la Edad de Bronce y el final del Neolítico. Se trata, manifiestamente, de la época de las ciudades sumerias en un lado del mundo, y los grandes asentamientos en Mesoamérica, en otro lugar de la geografía. Las primeras ciudades aparecen, y concomitantemente, también las primeras civilizaciones. En general el descubrimiento y el empleo del bronce produce, al mismo tiempo, una acumulación de conocimiento y un verdadero salto cualitativo en la producción de conocimiento.

La bibliografía sobre el primer momento es parcelada, altamente especializada en un plano o contexto, y por lo general se enfocan en alguno de los temas mencionados. No existe ningún trabajo de tipo general sobre la inflexión que constituye el año 3.000 a.e.v., aproximadamente. Somos nosotros quienes nos permitimos armar un tándem constituido por los elementos o piezas mencionadas. Quedaría para una lectura bastante más técnica vincular un plano geográfico con otro, y en cualquier caso, una lectura cuidadosa de la bibliografía permite un primer bosquejo sólido.

El segundo momento ha concentrado la mirada de la mayoría de los investigadores puesto que coincide exactamente con el comienzo de la civilización Occidental, el tránsito del mito al logos, el final de la Tiranía de los Treinta, el advenimiento de la democracia y los gobiernos de Solón y Pericles, y el paso de la Grecia arcaica a la Grecia clásica con tres clases de figuras: los sofistas, Sócrates como la filosofía oral y el método de la mayéutica, y Platón como el inicio de la filosofía Occidental. Sin la menor duda, se trata, narrado una y otra vez, del mito fundacional de la civilización occidental (al cual habría que agregar la síntesis misma que constituyen Atenas, Roma y Jerusalén).

Sin embargo, siempre ha quedado abierta la discusión acerca de las causas, las razones, y los factores del comienzo del pensamiento racional y abstracto y demás factores determinantes de y para la civilización occidental. En general, se trata de la aparición de un pensamiento abstracto, el tránsito de las matemáticas contables a la geometría euclidiana, una teoría incipiente de números, e incluso el descubrimiento anodino de los números irracionales. Asimismo, aparecen los primeros cimientos de la lógica, surge el concepto de causalidad que será absolutamente determinante en prácticamente toda la historia de Occidente, y emerge la filosofía que, más que la madre de las ciencias, connota la aparición del pensar abstracto por excelencia. En última instancia, verdaderamente determinante, el tránsito de la Grecia arcaica a la Grecia clásica significa el nacimiento de la idea más pura y fuerte de democracia, algo que irá a permear a todas las discusiones sobre el orden social humano en la historia subsiguiente.

El tercer momento tiene lugar gracias a la superación de los movimientos milenaristas y la emergencia de los Renacimientos: el primero -denominado genéricamente como "primer Renacimiento"-alrededor del año 1100 y su proyección -gradual- hasta el Quattrocento con sus grandes figuras y realizaciones. No hace mucho se ha escrito ya el poema del Beowulf, y es el momento cuando las Universidades de Oxford y de París comienzan las traducciones de Aristóteles al árabe y al latín, con las subsiguientes improntas de Pedro Abelardo, las múltiples Summas y Guillermo de Champeaux. Ulteriormente, ya en el Quattrocento propiamente dicho, se trata de los nombres de Miguel Angel, Leonardo Da Vinci y la historia subsiguiente. Los primeros cimientos del pensamiento científico moderno aparecen gracias a la obra de Ockham, Bacon, el propio Da Vinci y G. Bruno. Muy significativamente, tiene lugar el tránsito del sistema de numeración romano hacia el sistema de numeración arábico, además de la introducción del número cero en Occidente 
(descubierto en la India) gracias a los árabes, lo cual permite la invención o el descubrimiento del infinito, por primera vez en la historia de la humanidad. Por otra parte, aparece la lógica simbólica, y con los modernos burgos aparecerá una nueva clase social que habrá de tener una importancia singular en los últimos quinientos años de la historia de Occidente: la burguesía.

La bibliografía sobre cada uno de los tres períodos anteriores es amplia y creciente. Sin embargo, quisiéramos dirigir la mirada sobre el cuarto momento: en nuestros días, cuando emergen ciencias de frontera posibles a partir de problemas de frontera y que son acaso la mejor expresión de lo que queremos caracterizar aquí por en qué consiste pensar la complejidad. De manera muy significativa, el computador se convierte en este momento en un fenómeno cultural que al mismo tiempo que sirve de antecedente de las ciencias de la complejidad, también tiene influencia en la consolidación y desarrollo de las ciencias de la complejidad (Pagels 1989, Maldonado y Gómez 2015). De manera muy significativa, dicho con otras palabras, se trata del tránsito de la "pequeña ciencia" a la "gran ciencia" en la expresión propia que acuña originariamente De Solla Price (1986), y que no por coincidencia se corresponde con la emergencia de la sociedad de la información y el tránsito de ésta a la sociedad del conocimiento. Esto es, de la comprensión y el trabajo disciplinar hacia la constitución de fabulosos equipos científicos, académicos, administrativos y financieros que trabajan en torno a un tejido de problemas y sobre la base de intereses compartidos en toda la línea de la palabra.

Como quiera que sea, lo común a estos cuatro momentos radica en que encontramos un pensamiento horizontal, cruzado, inter, trans y multidisciplinario, así como prácticas integradas, diálogo de saberes, superación de la especialización, y un alto y refinado espíritu crítico y de cuestionamiento que ha prevalecido y gatillado esos momentos. Desde el punto de vista de la sociología del conocimiento, jamás había habido tantos científicos e investigadores y tantos ingenieros y técnicos como en nuestro tiempo, con el consiguiente crecimiento exponencial e hiperbólico del conocimiento.

En verdad, en el cuarto momento considerado, encontramos un grupo de ciencias que no tiene, por primera vez en la historia de la humanidad, un "objeto" de estudio. Este nuevo grupo de ciencias son ciencias como síntesis: las ciencias cognitivas, las ciencias de la salud, las ciencias de la vida, las ciencias de los materiales, las ciencias de la tierra, las ciencias del espacio, en fin, las ciencias de la complejidad.

De esta suerte, se trata de ciencias que se definen por problemas; por ejemplo, el conocimiento (cognition), la tierra, la salud, la vida, los materiales, el espacio o la complejidad, correspondientemente. Estos no son objetos de trabajo. Antes bien son problemas que es preciso elucidar, y para lo cual se requiere la confluencia de tradiciones antes distintas y hasta opuestas. Así, por ejemplo, en el caso de las ciencias cognitivas, ha dejado de ser evidente qué sea "conocimiento", para lo cual no se emplea ya el término de knowledge, sino el de cognition (un neologismo, también en inglés); ha dejado de ser evidente qué sea la vida, y es evidente que la biología, por ejemplo, es insuficiente para abordar y resolver el problema, y así sucesivamente. En cada uno de los casos, se trata de un problema en el que confluyen distintas metodologías, enfoques, disciplinas, ciencias y tradiciones; y al mismo tiempo, un problema que no puede ser resuelto, en absoluto, como fue quizás efectivamente el caso en el pasado en otros contextos, por una sola ciencia o disciplina. Pues bien, es justamente esto lo que caracteriza un problema de frontera. Quisiera subrayar esta idea: las ciencias de frontera son posibles a partir de la identificación de, y el trabajo con, problemas de frontera. En fin, una ciencia de frontera es ciencia como síntesis, y que se define semánticamente como ciencia en plural, en cada caso: "ciencias...". Un contraste notable con la idea clásica de "ciencia" (en singular). 


\section{Modo del pensar de la complejidad}

Digámoslo abiertamente: los enfoques cruzados, horizontales y transdisciplinarios no son, en absoluto, un patrimonio contemporáneo exclusivamente. La única diferencia, esa sí fundamental, es que por primera vez aquellos tienen lugar en un mundo diferente de suma cero. Todos los momentos anteriores sucedieron en un mundo de suma cero.

Esta sección no pretende ser exhaustiva, pero sí poner de relieve los rasgos distintivos, o clara y específicamente diferenciales, que permiten comprender qué significa pensar en el contexto de las ciencias de la complejidad.

Quiero sostener la idea según la cual pensar bien es pensar en todas las posibilidades y nadie piensa bien si no piensa con las matemáticas y/o las lógicas, algo que en general en la comunidad de complejólogos, en sentido amplio, no ha sido tenido en cuenta suficientemente. Más radicalmente, pensar en todas las posibilidades implica incluso considerar lo imposible; esto es, en estructuras, dinámicas, formas y comportamientos imposibles. Pues bien, existe un capítulo reciente, notablemente desde las matemáticas, que hace de lo imposible, por así decirlo, un tema propio de estudio. Sin embargo, estas ideas exigen una aclaración.

Los fenómenos, comportamientos y sistemas complejos son alta y crecientemente contraintuitivos (McCabe 2014). Por consiguiente la lógica formal clásica no sirve para estudiarlos ni comprenderlos. En el mismo sentido, como es sabido, los fenómenos de complejidad creciente están marcados por la flecha de la irreversibilidad del tiempo, son por tanto no-ergódicos, y esencialmente probabilísticos. Dos son los referentes sin los cuales, en absoluto, cabe pensar, por consiguiente, a la complejidad, a saber: la teoría de la evolución y la teoría cuántica. En otro momento me he ocupado de justificar esta dúplice necesidad.

Quiero defender la idea según la cual los sistemas complejos no-lineales son esencialmente discretos y al mismo tiempo permiten y demandan otras lógicas diferentes a la lógica simbólica o la lógica de predicados. Afortunadamente disponemos de estas "herramientas", por así decirlo, algo que en general en la comunidad de complejólogos no es muy conocido.

\subsection{Las matemáticas de sistemas discretos}

Cabe distinguir dos clases de matemáticas, así: las matemáticas de sistemas continuos y las matemáticas de sistemas discretos. Digamos de pasada que, por tanto, es un error creer que hay matemáticas cuantitativas y cualitativas (un mal chiste cuando se lo ve con los ojos del conocimiento). Las matemáticas de sistemas continuos trabajan esencialmente con estadística (descriptiva, inferencial, etc.), álgebra, cálculo (integral y diferencial), función o funciones, con el concepto de límite y con problemas de optimización. No es sobre este plano que queremos concentrar la mirada.

Por el contrario, las matemáticas de sistemas discretos trabajan con conjuntos parcialmente ordenados, conjuntos extremos, geometría discreta y combinatoria, con teoría discreta de probabilidades, con problemas combinatorios también llamados genéricamente como complejidad combinatoria, con topología, teoría de juegos y teoría de la decisión racional, con algunas de la lógicas no-clásicas, las matemáticas en general de sistemas computacionales, grafos e hipergrafos, así como con teselados. 
Pues bien, más exactamente, las matemáticas de sistemas discretos son las matemáticas de la complejidad en toda la acepción de la palabra. Dicho en términos precisos, todas las matemáticas de punta en la ciencia de frontera en el mundo son matemáticas de sistemas discretos.

En efecto, pensar la complejidad consiste en pensar, por primera vez en la historia de la humanidad no única y no principalmente en tendencias o en actualidades, sino además y fundamentalmente en rupturas, quiebres, discontinuidades, sorpresas e irrupciones. Estos conceptos se han expresado en la jerga de complejidad como "autoorganización", "fluctuaciones", "turbulencias", "inestabilidades", "incertidumbre" y "emergencia", "bifurcaciones", por ejemplo. Para decirlo de manera puntual, se trata de sistemas con valores distintos y separados. Y las herramientas exactas de estudio han llamado la atención sobre transiciones de fase de primero y segundo orden (Solé 2011), puntos y estados críticos, criticalidad autoorganizadas y distribuciones en o como leyes de potencia. Un estupendo manejo del lenguaje es una condición necesaria en el desarrollo científico, pero, al mismo tiempo, el lenguaje no debe disimular los fenómenos mismos. En este caso, los fenómenos son, quiero decirlo de manera franca, discretos. Un sistema se dice que es discreto cuando tiene un número finito, aunque muy grande, de estados contables. Sociológica y culturalmente hablando, por tanto, las matemáticas de sistemas discretos corresponden a las matemáticas de un mundo diferente de suma cero.

\subsection{Las lógicas no-clásicas}

Concomitante o complementarias con las matemáticas de sistemas discretos, pensar la complejidad exige, a la vez, de un sólido manejo de lógica. Nadie puede pensar sin lógica, pero las lógicas propias de los sistemas complejos no-lineales son las lógicas no-clásicas. Esto es, más exactamente, las lógicas adecuadas a sistemas esencialmente variables, impredecibles, marcados por el tiempo y que admiten escalas diferentes son las lógicas no-clásicas (Weingartner 2010), Ilamadas en ocasiones igualmente como lógicas filosóficas (Goble 2005).

Las lógicas no-clásicas emergen debido a una dúplice condición: o bien debido a la laxitud, o bien a un rigor extremo de la lógica formal clásica (específicamente, cuando se trabaja en términos de cuantificación, un tema técnico que quisiera dejar aquí de lado). Como es sabido, la lógica formal clásica es la misma lógica simbólica, la lógica matemática, la lógica proposicional o lógica de predicados -cuatro maneras diferentes de designar un solo y mismo ámbito-, la cual si bien encuentra sus orígenes remotos en Aristóteles, en realidad poco y nada tiene ya que ver con la tradición aristotélica, en la acepción más amplia de la palabra.

En el panorama de las lógicas no-clásicas encontramos la lógica del tiempo, la lógica deóntica, la lógica epistémica, las lógicas modal y multimodal, la lógica de contrafácticos, la lógica abductiva, la lógica condicional, la lógica dinámica, la lógica de la relevancia, la lógica intuicionista, la lógica de fibras, las lógicas difusa y polivalentes, la lógica probabilística y la lógica cuántica, la lógica paraconsistente, y la lógica libre, entre otras. Se trata de una dimensión reciente, vital y en constante nacimiento y desarrollo.

Para decirlo de manera sucinta, a la diversidad y complejidad creciente del mundo y la naturaleza le corresponde un pluralismo lógico; esto es, de sistemas de verdad y sistemas de cuantificación altamente sugestivos, tanto como una multiplicidad de sistemas deductivos. De la misma manera que la complejidad implica el no-reduccionismo, asimismo el trabajo con las lógicas no-clásicas demanda expresamente una crítica a un sistema lógico determinado -en cualquier sentido o aspecto. 
Por consiguiente, es supino pretender cualquier pelea o discusión con la lógica, primero como si hubiera un solo sistema lógico, y segundo porque, à la lettre, el trabajo con complejidad es exactamente el trabajo con pensamiento abstracto, y la lógica, tanto como las matemáticas o la filosofía, constituyen los modos por excelencia de un pensar abstracto. $Y$ en segundo lugar, debido a que justamente nos encontramos en conocimiento de frontera, o bien porque las ciencias de la complejidad son ciencia de lo posible, antes que de lo real (Maldonado 2014a). Y el trabajo con lo posible ha sido, de manera tradicional el ámbito del arte, las matemáticas, la filosofía, las lógicas y la música en el sentido excelso de la palabra. Para no hablar acerca del propio aprendizaje de un idioma extranjero, que es el aprendizaje con, de posibilidades.

En otras palabras, mientras que la ciencia normal es ciencia de lo real-en cualquier acepción-, las ciencias de la complejidad son ciencia de lo posible. Esta idea se entiende mejor con la consideración que sigue a continuación.

\subsection{Espacios de fase e imposibilidad}

Como es sabido, las posibilidades se denominan en el lenguaje de la complejidad espacios de fase. Estos son espacios imaginarios, no reales en el sentido empírico de la palabra, en los cuales, de manera puntual, se identifican estados y puntos críticos. Por derivación, el estudio de la criticalidad de un fenómeno o sistema entraña considerar la subcriticalidad y supracriticalidad de los mismos. Esto es, los estados o momentos en los que aún no existe irreversibilidad o bien en los que la irreversibilidad de un estado ya es inevitable. Los espacios de fase son momentos en los que suceden bifurcaciones, cambios cualitativos, en fin, cambios en las trayectorias (= historias) de un fenómeno determinado. Estos espacios no se ven, literalmente, con los ojos; por el contrario, se los construye, se los concibe. En este sentido, una herramienta dúctil de ver la posibilidad es el computador y la computación. De aquí el significado y la impronta del modelamiento y la simulación en el trabajo con los sistemas de complejidad creciente.

Quisiera dirigir la mirada hacia el extremo, por así decirlo, en el estudio de las posibilidades. Se trata de la cohomología, cuyo ámbito específico de trabajo se denomina las multiplicidades. Un tema matemáticamente muy sofisticado y, sin embargo, bastante natural.

Una multiplicidad es en matemáticas la cantidad de pertenencias de un miembro de un multiconjunto. En otras palabras, una multiplicidad es un espacio topológico que en escala micro, en los aspectos singulares, se asemeja a un espacio euclidiano, pero globalmente difiere por completo. En términos elementales: a escala micro puede ser considerado como una figura plana euclidiana (líneas, planos, círculos), pero a escala global, esto es, como un todo, dista mucho de ser un espacio euclidiano.

El padre de la cohomología en general y del que es quizás el capítulo más importante que es la cohomología de gavilla (sheaf cohomology) es el matemático francés Alexander Grothendieck (19282014). Algunos de los desarrollos más recientes en el tema corresponden a R. Penrose, quien ha trabajado justamente en la cohomología de figuras imposibles.

Ahora bien, vale recordar que las tres operaciones básicas que se hacen con los objetos o con el espacio en topología son: torcer, estirar y comprimir. Derivativamente, existen funciones y tensores de torsión y demás, correspondientemente.

La manera más básica de entender y de acercarse a la cohomología consiste en recordar que en matemáticas la teoría de homologías -que remiten ulteriormente a los grupos abelianos (en honor del 
matemático noruego N. H. Abel)- se encarga del estudio de grupos (o módulos) de acuerdo a un espacio topológico. Más exactamente, la homología contribuye a la clasificación de los tipos de espacios.

Pues bien, el aspecto verdaderamente apasionante es que existen, en matemáticas, infinitos espacios. Y cada geometría designa un espacio distinto, y más radical y exactamente, un mundo distinto. Así, tenemos la geometría euclidiana, las geometrías no euclidianas (Riemann y Lobachevsky), la geometría proyectiva, la geometría de taxis, la geometría hiperbólica, la geometría de fractales, y así muchísimas más.

Al respecto, es fundamental observar que en el universo y en la naturaleza coexiste una multiplicidad de espacios diferentes. Y entre ellos, hay incluso espacios imposibles, formas y patrones imposibles, estructuras y comportamientos imposibles. Pues bien, la cohomología consiste en el estudio de grupos (abelianos) definidos a partir del estudio de co-cadenas, cociclos o cobordes (vale recordar que la teoría de catástrofes, desarrollada por R. Thom, nace a partir de los antecedentes de trabajo por parte del propio Thom en el tema del cobordismo).

Como quiera que sea, pensar la complejidad equivale exactamente a pensar en posibilidades y en sus modalidades o modalizaciones, lo cual incluye e implica pensar incluso en lo imposible, como una tematización explícita y consciente. Como se aprecia el contraste con la historia clásica de la ciencia y la filosofía no puede ser más agudo.

\section{Consecuencias e implicaciones de un pensar complejo}

Pensar la complejidad tiene múltiples consecuencias en los órdenes teórico, práctico, social, político y existencial. Quisiera aquí, por razones de espacio, presentarlas de manera somera a fin de reflexionar acerca de las implicaciones de un pensar complejo.

Nos encontramos, manifiestamente, en medio de una auténtica revolución científica; por decir lo menos, en el sentido kuhniano de la palabra. Las revoluciones científicas han sido presentadas como el esfuerzo de solución a anomalías en el paradigma dominante; en nuestro caso, en la corriente principal de la ciencia, la filosofía y la cultura. Esto implica que, en buena ciencia, los complejólogos no trabajamos a partir de campos, temas, áreas y ni siquiera preguntas. Por el contrario, más exactamente, los complejólogos pensamos a partir de (la identificación de) problemas. Ahora bien, cuando existe un problema, son tres las vías de resolución de los mismos: por vía de cálculo, esto es, notablemente, mediante el trabajo con algoritmos; por vía de razonamientos; o bien por vía de planteamiento y solución de ecuaciones (Dowek 2011).

En el primer caso, la comunidad de complejólogos ha hecho de las metaheurísticas un campo propio de trabajo (Talbi 2009) mediante el cual los más difíciles y apasionantes problemas de complejidad computacional son planteados y resueltos. Con una observación importante: la resolución no es ya, por primera vez en la historia de la humanidad, planteada en términos de soluciones exactas y precisas. La bibliografía al respecto es amplia y creciente. En el segundo caso, se trata, manifiestamente del recurso a la imaginación como a la forma misma sin la cual nadie puede llamarse a sí mismo investigador o científico. De manera general, cabe decir que los grandes avances en el conocimiento se han fundado en experimentos mentales, antes que el llamado a la formulación de hipótesis, observación, descripción, y demás. La imaginación entra en complejidad a través de dos caminos distintos: el modelamiento y la simulación, y las lógicas no-clásicas (Beal y Restall 2006). La imaginación es adecuadamente llamada aquí también como pompas de intuición. Finalmente, en el tercer caso, se trata del reconocimiento de que la 
mejor manera de resolver un problema es innovando, y que el desplazamiento del foco del problema exige una actitud de total radicalidad. Es posible traducir esta idea recurriendo a Einstein cuando sostenía: la solución a un problema no se puede dar al interior del marco en el que surge el problema.

Esto quiere decir que cuando se quiere efectivamente resolver por lo menos un problema, lo mejor que cabe hacer es transformar el marco-social, semántico, cultural, económico, lógico, epistemológico, en fin, político- en el que surge el problema. De lo contrario no se habrá resuelto nada. Una "investigación epidemiológica" es el eufemismo para esa clase de problemas que se formulan pero que ulteriormente quedan sin resolver de manera efectiva, real o radical; o bien de aquellos problemas que se formulan porque ya se conoce su solución.

Quisiera, en consonancia con este texto llevar el argumento, finalmente, algo más lejos.

Pensar en complejidad significa no analizar. El pensamiento analítico -y todas sus expresiones y herramientas- comprenden al mundo en términos de desagregación, división, fragmentación. Que son, desde luego, aproximaciones perfectamente legítimas, a condición de que se entienda que los sistemas complejos no se pueden analizar. Por el contrario, más exactamente, los sistemas, fenómenos y comportamientos complejos demandan y facultan a la vez para algo que la cultura occidental no nos ha capacitado en absoluto, a saber: pensar de forma sintética.

Digamos en general que existen síntesis proteicas, el anabolismo, síntesis por sexualidad, síntesis granular, síntesis de la imaginación, síntesis de la percepción, y muchas otras más. Una de las formas más conspicuas de avance del conocimiento en la historia de la ciencia, la filosofía y el arte consiste justamente en aquellos nombres, momentos y lugares en los que se han configurado grandes síntesis. Lo restante son predominios de Maquiavelo; de Maquiavelo o Aristóteles; de ellos, o de pensamiento estratégico y control. Eso es, divide y reinarás (vale recordar que quien descubrió y sistematizó el análisis como estructura fundamental del pensamiento fue Aristóteles).

Dada la limitación de espacio aquí, quiero subrayar que el pensar la complejidad no es único ni canónico (Maldonado 2014b). No existe una canónica en el pensar la complejidad; derivativamente, resulta un contrasentido hablar de "el método" en este contexto. No hay una canónica ni en el pensamiento complejo -en cualquiera de sus matices-, ni en las ciencias de la complejidad. Al fin y al cabo, la complejidad no es una cosmovisión: es un problema. En sentido riguroso, no se piensa en función de la complejidad, en cuyo caso se convierte el lenguaje y los autores y los textos en una doctrina. Por el contrario, pensamos la complejidad misma -allí cuando, donde y como acaece. Pues no es ni bueno ni necesario ni deseable que todas las cosas sean complejas. En numerosas ocasiones es incluso deseable que no sea así.

Por el contrario, cuando un sistema lineal se convierte en uno no-lineal, cuando un fenómeno predecible se torna impredecible, cuando un sistema rígido se comporta en el filo del caos, por ejemplo, entonces los complejólogos: a) tenemos la palabra y tenemos la obligación moral e intelectual de decir cosas significativas; b) podemos contribuir en algo al mundo; esto es, muy exactamente, a hacer posible la vida en general y cada vez más posible. Pero mientras el mundo no exhiba complejidad o los sistemas de control muy diversos -rígido, piramidal, distribuido, paralelo o difuso- aún sean posibles, aún no será el tiempo o el momento de la complejidad.

Lo anterior no obstante, se hace indispensable precisar una cosa. Los complejólogos trabajamos en tiempos y en contextos de complejidad, según la siguiente distinción, que es meramente epistemológica. 
La complejidad trabaja con fenómenos, sistemas, tiempos y comportamientos de crisis, según si: a) la crisis ya está presente y es inminente, b) la crisis aún no ha llegado pero podría llegar a suceder y c) trabajamos con crisis incluso aunque jamás lleguen a tener lugar.

Lo anterior significa que, en contraste con la ciencia y el pensamiento normales, lo propio de complejidad no consiste en hacer predicciones retrospectivas. Esto es, explicar los acontecimientos una vez que han sucedido y de acuerdo al modo como tuvieron lugar, llegando incluso, in extremis, a explicar por qué debían y podían haber sucedido. Una predicción retrospectiva es lo más trivial que se pueda hacer en buena ciencia y pensamiento.

Es posible hacer una paráfrasis del concepto mencionado de crisis. Si se quiere, para decirlo solo de una manera rápida y en el vocabulario en boga, el trabajo con complejidad es el trabajo mismo con situaciones y momentos de riesgo (Helbing 2013). Un grupo de complejidad sería algo así como un comité de crisis en el sentido señalado, y en cualquier ámbito que se prefiera.

En este sentido, para decirlo de manera franca y directa: las ciencias de la complejidad no son ciencia de lo real; sino, mucho mejor aún, ciencia de lo posible. El modo como trabajamos es entonces, en el contexto cultural que vivimos, mediante el modelamiento y la simulación; en otras palabras, con la formulación de modelos, teorías, y demás (Kaye 2007), reconociendo explícitamente lo siguiente: el trabajo con modelamiento y simulación implica el manejo y el conocimiento, la familiaridad y el dominio del computador y los temas y problemas propios de la computación; esto es, de programación y el poder expresivo de un lenguaje. Con la advertencia explícita de que el computador y la computación no implican, en absoluto, un reduccionismo tecnológico o algo semejante. El computador es un artefacto cultural, un fenómeno cultural. Y es de tal tipo que, literalmente, la calidad de la educación o la calidad de vida de las personas atraviesa medularmente por el tipo de conocimiento, familiaridad y experticia de la tecnología que se tiene. Este argumento se puede ilustrar fácilmente en ámbitos como la medicina, la educación, las políticas públicas, o en la investigación de punta en ciencia.

Sin pensar, en absoluto, en términos de causalidad, cabe decir que cuanto mayor y mejor es la tecnología de que se dispone, tanto mayor y mejor es la calidad de vida de un individuo, un grupo familiar, una sociedad o una nación, por ejemplo.

En otras palabras: la más grande de todas las brechas generacionales hoy por hoy es la brecha tecnológica. Mientras que los más jóvenes tienen una relación de familiaridad hacia las nuevas tecnologías, sus ritmos y procesos, los mayores parecen ser reacios y reactivos, sospechosos y algo lerdos con respecto al manejo de la tecnología; esto es, muy puntualmente, de los sistemas tecnológicos y computacionales actuales y que nos rodean, nos moldean y contribuimos a moldear. Las revoluciones científicas, análogamente a las revoluciones políticas o sociales, son jalonadas por los sectores más juveniles, críticos o pensantes de la sociedad. Jalonadas, pero no se pueden reducir jamás únicamente a ellos.

\section{Conclusiones}

Nadie piensa sin la cabeza. Pero las cosas verdaderamente importantes no se piensan con la cabeza. Por el contrario, implican al cuerpo mismo, y nos arrastran como una totalidad indivisa. Son tan solo las pequeñas decisiones, esas que en lógica y matemáticas se denominan triviales, las que demandan el trabajo del cerebro. 
Pensar, por lo demás, no es un acto voluntario o deliberado. Nadie piensa porque quiere. A algunos les acaece pensar; a otros no. Pensar, definitivamente, es perfectamente distinto a conocer, incuso en aquella perspectiva original y radical de Maturana y Varela cuando afirman, con razón, que el conocimiento y la vida son idénticos, o una sola y misma cosa.

No sin razones, entre la comunidad de complejólogos aquello que puede acercarse o asimilarse a un pensar complejo ha sido expuesto de manera negativa. Así por ejemplo, se trata de un pensar noalgorítmico, lo cual, literalmente, significa el no acatamiento de leyes, reglas, normas, preceptos, mandamientos o cánones de ningún tipo. En otros lugares me he ocupado de este tema (Maldonado 2014b), específicamente en relación con el cruce entre biología y complejidad. Otras caracterizaciones semejantes y próximas han sido recurrentes en la bibliografía: así por ejemplo, no reduccionismo, nodeterminismo, y demás.

Pensar la complejidad no es un acto o un proceso necesario, evidente ni inmediato. Pensamos la complejidad al cabo; es decir, luego de un arduo y serio trabajo en el que se mezclan innovación y sospecha, crítica y creatividad, sensaciones personales y sociales conjuntamente con situaciones límite en sentido amplio en el que la emocionalidad está plenamente incorporada o inmiscuida. Nadie parte de la complejidad: llegamos a ella. Pero entonces asistimos al comienzo de otra historia.

\section{Bibliografía}

Beal, J. C. y Restall, G. 2006. Logical pluralism. Oxford: Clarendon Press.

De Solla Price, D. 1986. Little science, big science. New York: Columbia University Press.

Dowek, G. 2011. Les métamorphoses du calcul. Paris: Le Pommier.

Goble, L. 2005. The Blackwell guide to philosophical logic. London: Blackwell Publishing.

Helbing, D. 2013. Globally networked risks and how to respond. Nature 497: 51-59.

doi:10.1038/nature12047

Kaye, R. 2007. The mathematics of logic. A guide to completeness theorems and their applications.

Cambridge: Cambridge University Press.

Lévêque, P. 2012. Las primeras civilizaciones. De los despotismos orientales a la ciudad griega. Madrid: Akal.

Maldonado, C. E. y Gómez, N. 2015. Biological hypercomputation: a new research problem in complexity theory. Complexity 20(4): 8-18. doi: 10.1002/cplx.21535

Maldonado, C. E. 2014a. Reflexión sobre las implicaciones políticas de la complejidad. Alpha 38: 197-214. doi: 10.4067/S0718-22012014000100013

Maldonado, C. E. 2014b. ¿Qué es un sistema complejo? Revista Colombiana de Filosofía de la Ciencia 14(29): 71-93. 
McCabe, V. 2014. Coming to our senses. Perceiving complexity to avoid catastrophes. Oxford: Oxford University Press.

Pagels, H. 1989. Los sueños de la razón. El ordenador y los nuevos horizontes de las ciencias de la complejidad. Barcelona: Paidós.

Roberts, J. M. 2010. Historia del mundo. De la prehistoria a nuestros días. Madrid: Debate.

Talbi, E-G. 2009. Metaheuristics: From design to implementation. New Jersey: Wiley \& Sons.

Solé, R. 2011. Phase transitions. Princeton \& Oxford: Princeton University Press.

Weingartner, P. 2010. Alternative logics. Do sciences need them. Verlag: Springer.

Recibido el 3 Jul 2015

Aceptado el 16 Sep 2015 